\title{
Características da Carcaça e da Carne de Novilhas Búfalas Terminadas em Confinamento, em Função do Uso de Promotor de Crescimento ou de Esferas de Chumbo no Útero ${ }^{1}$
}

\author{
Ivanor Nunes do Prado ${ }^{*}$, Jair de Araújo Marques ${ }^{3}$, José Luiz Moletta ${ }^{4}$, Lívia Maria Araújo \\ Macedo ${ }^{5}$, Ivor Martin do Prado ${ }^{6}$, Juliana Martin do Prado ${ }^{7}$, Nilson Evelázeo de Souza ${ }^{8}$, Jesuí \\ Vergílio Visentaner 8
}

\begin{abstract}
RESUMO - Objetivou-se com este trabalho avaliar o efeito da simulação da prenhez (introdução de 100 esferas de chumbo no útero - CHU) e do uso de promotor de crescimento (PRC), em comparação a novilhas testemunhas vazias, porém, com atividade ovariana (novilhas vazias - VAZ) sobre o peso de carcaça, a conformação da carcaça, os comprimentos de carcaça e de perna, as espessuras do coxão e da gordura de cobertura, as proporções de osso, músculo e gordura e as perdas ao descongelamento e à cocção. Foram utilizadas 20 novilhas bubalinas da raça Murrah com média de 20 meses de idade. Após período de confinamento ( 84 dias), foram registrados pesos médios ao abate (PVA) de $422,6 \mathrm{~kg}$, para as novilhas do tratamento VAZ; 414,9 kg, para as do tratamento CHU; e $451,8 \mathrm{~kg}$, para as do tratamento PRC. Não houve diferença entre tratamentos para o peso vivo ao abate $(429,8 \mathrm{~kg})$, peso de carcaça quente (209,0 kg), conformação da carcaça (8,2 pontos), comprimentos de carcaça $(122,9 \mathrm{~cm})$ e de perna $(68,1 \mathrm{~cm})$, espessuras do coxão $(25,0$ $\mathrm{cm})$ e da gordura de cobertura $(4,8 \mathrm{~mm})$, área de olho de lombo $\left(57,1 \mathrm{~cm}^{2}\right)$, proporções de músculo $(60,6 \%)$, de osso $(18,9 \%)$ e de gordura (20,5\%), relações músculo/osso (3,2), músculo mais gordura (porção comestível)/osso (4,3), perdas ao descongelamento ( $9,3 \%$ ), à cocção $(23,4 \%)$ e do congelado após a cocção ( $30,5 \%)$, maciez ( 7,8 pontos), suculência ( 7,0 pontos) e palatabilidade ( 6,8 pontos). Concluiu-se que a utilização de promotor do crescimento e de esferas de chumbo no útero não influenciou as variáveis analisadas.
\end{abstract}

Palavras-chave: composição física, coxão, cozimento, maciez

\section{Effects of Growth Promoter or Lead Spheres Placed in the Uterus on Carcass and Meat Traits of Buffalo Heifers Finished in Feedlot}

\begin{abstract}
This study was conducted to evaluate the effects of lead spheres placed in the uterus to simulate pregnancy and of growth promoter on carcass weight, carcass conformation, carcass and leg lengths, beef round and fat thickness, percentages of bone, muscle, and fat, and thawing and cooking losses. Twenty Murrah buffalo heifers averaging 20 months of age were randomly assigned to one of three treatments: control (non-pregnant heifers but with ovarian activity - NP), lead (100 lead spheres placed in the uterus - L), or growth promoter (GP). After 84 days in feedlot the average slaughter weights were 414.8, 451.8, and 422.6 kg for heifers on NP, L, and GP treatments, respectively. No significant differences were observed among treatments for slaughter body weight (429.8 kg), hot carcass weight (209.0 kg), carcass conformation (8.2 points), carcass length $(122.9 \mathrm{~cm})$, legs length $(68.1 \mathrm{~cm})$, cushion thickness $(25.0 \mathrm{~cm})$, loin eye area $\left(57.1 \mathrm{~cm}^{2}\right)$, fat thickness $(4.8 \mathrm{~mm})$, percentages of muscle $(60.6 \%)$, bone (18.9\%), and fat (20.5\%), muscle/bone ratio (3.2), muscle plus fat/bone ratio (4.3), thawing loss (9.3\%), cooking (23.4\%) and freezing to cooking losses (30.5\%), tenderness (7.8 points), juiciness (7.0 points), and flavor (6.7 points). It was concluded that the use of growth promoter or lead spheres in the uterus had no significant effects on the studied variables.
\end{abstract}

Key Words: beef round, cooking, physical composition, tenderness

\section{Introdução}

O rebanho de bubalinos no mundo apresentou crescimento de 50\% de 1978 a 1990 (Mattos, 1992). No entanto, segundo dados do ANUALPEC (2003), a população bubalina no Brasil é de aproximadamente 1.150 .052 animais. Na última década, esta população apresentou crescimento de 23,6\%, aumentando de 930.134 cabeças em 1994 para os números atuais. Todavia, neste mesmo período, este crescimento foi

\footnotetext{
${ }^{1}$ Parte da tese do segundo autor - Programa de Pós-Graduação em Zootecnia (PPZ) da Universidade Estadual de Maringá (UEM).

2 Professor Titular do Departamento de Zootecnia da Universidade Estadual de Maringá - Av.: Colombo, 5790 CEP: $87020-900$ MaringáPR. Bolsista Produtividade I-CNPq.

${ }^{3}$ Doutor EMATER/IAPAR. Professor do Integrado Colégio e Faculdade Campo Mourão - PR.

${ }^{4}$ Pesquisador do IAPAR.

${ }^{5}$ Aluna de Especialização do PPZ/UEM. Bolsista de Aperfeiçoamento Técnico-CNPq.

${ }^{6}$ Aluno da Graduação do curso de Engenharia de Alimentos da UEM.

7 Aluna da Graduação do curso de Engenharia de Alimentos da UEM. Bolsista CNPq.

${ }^{8}$ Professor Doutor do Departamento de Química da UEM.

* E.mail para contato: inprado@uem.br
} 
de 7,7\% no estado do Paraná, sendo bem mais modesto que o crescimento nacional. No entanto, deve ser ressaltada a adaptação da espécie bubalina aos trópicos, podendo-se realizar sua exploração racional de norte a sul do Brasil.

A carne de bubalinos, com menor quantidade de gordura saturada e colesterol total (Morgan, 1993), pode atender à demanda por este tipo de produto. Além disso, pesquisas realizadas demonstraram que os bubalinos apresentam grande produtividade e capacidade de adaptação às condições brasileiras (Nogueira et al., 1989; Baruselli et al., 1993; Oliveira et al., 1994; Villares, 1994; Jorge et al., 1997). Desta forma, a bubalinocultura passará a ser uma atividade atraente para produção de carne, tanto em pastagens, como mostra Macedo et al. (2001), quanto em confinamento, como demonstra Jorge \& Fontes (2001).

$\mathrm{O}$ rendimento de carcaça e de cortes comerciais e o peso de carcaça são medidas de interesse dos frigoríficos (Berg \& Butterfield, 1976). Segundo Costa et al. (2002), o abate de animais com pesos mais elevados pode resultar em mudança das taxas de desempenho e características de carcaça. Portanto, o peso de abate tem grande importância na terminação de animais confinados, pois altera o custo e a qualidade da carne produzida, como demonstrado por Forrest et al. (1979) e Rodrigues et al. (2003).

Outro parâmetro importante na avaliação de carcaças é a musculosidade, que pode ser avaliada pela conformação da carcaça (Rodrigues et al., 2001; Jorge et al., 2003a,b; Vaz et al., 2003).

De modo geral, as características físicas da carcaça podem ser manipuladas por meio do manejo nutricional (Marques et al., 2000), da idade de abate (Restle et al., 1999), da escolha de raças para produção de carne (Moletta \& Restle, 1996), do sistema de produção a ser utilizado, entre outros fatores.

Um dos fatores que podem proporcionar melhor desempenho aos animais e produção de carne com maior rapidez são os hormônios, normalmente aplicados na forma de um pequeno pelete, via implante subcutâneo, na orelha dos animais, agindo como promotor de crescimento. A função dos promotores de crescimento estrogênicos é melhorar o desempenho e a rentabilidade da atividade, como demonstrado em trabalhos realizados nos Estados Unidos (Rumsey et al., 1999a; Rumsey et al., 1999b; Rumsey et al., 1999c) e no Brasil (Pádua et al., 2001). Essa prática, apesar de largamente utilizada nos Estados Unidos, no
Brasil é proibida, sob a alegação que a utilização desses produtos pode deixar possíveis resíduos na carne. Todavia, Ferreira et al. (2001), avaliando a presença de resíduo de acetato de trembolona na urina, observaram que, a partir de 65 dias após a aplicação, não foi mais detectado resíduo pelo teste ELISA, na urina de animais tratados. Hoffmann (1998) registrou níveis semelhantes de progesterona nos tecidos (músculo, fígado, rim e gordura) entre animais tratados e não-tratados com estradiol + progesterona. Esse autor também observou níveis de testosterona, nos mesmos tecidos, superiores em touros quando comparados com novilhos tratados com estrógeno + testosterona.

$\mathrm{O}$ aparecimento de estro pode prejudicar o desempenho de fêmeas confinadas (Prado et al., 2000), assim como animais abatidos no dia do cio podem apresentar alterações na qualidade da carne, como carne DFD (escura, dura e seca) e hematomas (Crowe et al., 1995). Desta forma, a utilização de produtos ou técnicas que evitem o cio tem sido estudada por pesquisadores e produtores e, entre estas opções, está a aplicação de esferas de chumbo no interior do útero. Marques et al. (2001), ao utilizarem esta técnica em novilhas, obtiveram desempenho superior naquelas tratadas em relação às vazias.

Em razão do pequeno número de trabalhos realizados no Brasil comparando formas de terminação de fêmeas de descarte para produção de carne, foram avaliados neste estudo parâmetros qualitativos e quantitativos da carcaça de búfalas com implante de promotor de crescimento ou de esferas de chumbo no útero terminadas em confinamento.

\section{Material e Métodos}

Este trabalho foi realizado na Fazenda Experimental de Iguatemi, no Setor de Bovinocultura de Corte, pertencente à Universidade Estadual de Maringá.

Foram utilizadas 20 búfalas, distribuídas em três tratamentos: VAZ - novilhas com atividade ovariana (sete búfalas); CHU - novilhas com 100 esferas de chumbo (esterilizadas a álcool $-96^{\circ}$ ) e introduzidas no corno uterino, com auxílio de uma pipeta de inseminação artificial (sete búfalas); e PRC - novilhas com implante de promotor de crescimento (20 $\mathrm{mg}$ de benzoato de estradiol e $200 \mathrm{mg}$ de testosterona - Synovex $\mathrm{H}^{\circledR}$ ) (seis búfalas).

As búfalas foram pesadas $(350,5 \mathrm{~kg} \pm 39,8)$, identificadas com brinco plástico na orelha direita, 
vacinadas contra febre aftosa $\left(\right.$ Rhodia $\left.^{\circledR}\right)$, vermifugadas com $8 \mathrm{~mL}$ de vermífugo, à base de ivermectina (Ivomec ${ }^{\circledR}$ ) e alojadas em três piquetes de $270 \mathrm{~m}^{2}$ com pouca arborização. Para melhorar o conforto térmico dos animais, foi disponibilizada uma cobertura de sombrite para cada piquete de $20 \mathrm{~m}^{2}$. Cada piquete dispunha de $6 \mathrm{~m}$ lineares de comedouro de concreto e bebedouro com bóia de vazão total e capacidade de $250 \mathrm{~L}$.

A ração completa (silagem de cana-de-açúcar $+1 \%$ uréia, milho em grão quebrado + farelo de soja + sal mineral) (Tabela 1) foi fornecida ad libitum duas vezes ao dia (às 8 e 16 h). A ração foi calculada para proporcionar ganho de peso vivo médio de 1,0 kg/animal/dia, segundo NRC (1996) para bovinos de corte.

Foi realizada palpação retal nas 20 búfalas 30 dias antes do início do experimento, para avaliar as condições reprodutivas dos animais. Após o período de adaptação (30 dias), foi iniciada a sincronização de cio pelo protocolo "Ovsynch", conforme descrito por Pursley et al. (1995). Foram aplicados, no dia zero, $2 \mathrm{~mL}$ de Conceptal ${ }^{\circledR}(\mathrm{GnRH}-200 \mu \mathrm{g})$, sete dias após, $2 \mathrm{~mL}$ de Preloban ${ }^{\circledR}\left(\mathrm{PGF}_{2} \alpha-150 \mu \mathrm{g}\right)$ e, dois após esta aplicação, $2 \mathrm{~mL}$ de Conceptal ${ }^{\circledR}(\mathrm{GnRH}-200 \mu \mathrm{g})$. Dezesseis horas após o término deste protocolo, procedeu à colocação das esferas de chumbo nas búfalas do tratamento CHU e do promotor de crescimento nas búfalas do tratamento PRC. Para colocação do chumbo, as búfalas foram contidas e o procedimento foi semelhante ao da inseminação artificial. A diferença dos métodos foi que, ao passar com a pipeta através da cérvice, a bainha foi direcionada para a extremidade cranial de um dos cornos uterinos. As esferas contidas na bainha foram impulsionadas utilizando-se um expansor. Foram colocadas 100 esferas de chumbo de $4 \mathrm{~mm}$ de diâmetro, esterilizadas previamente com álcool $96^{\circ}$. No mesmo dia, foi implantado o promotor de crescimento na orelha direita das búfalas do tratamento PRC. O implante continha $200 \mathrm{mg}$ de testosterona e $20 \mathrm{mg}$ de benzoato de estradiol (Synovex $-\mathrm{H}^{\circledR}$ ).

Após período de confinamento (84 dias), as novilhas apresentaram PVA médios de 422,6 kg $\pm 43,0$ (tratamento VAZ), 414,9 kg $\pm 41,1$ (tratamento CHU) e 451,8 kg $\pm 26,3$ (tratamento PRC). No momento do abate dos animais, as carcaças foram identificadas, pesadas e conduzidas para câmara de resfriamento à temperatura interna de 1 a $5^{\circ} \mathrm{C}$, onde permaneceram durante 24 horas. Após o resfriamento, utilizou-se o lado direito da carcaça para avaliar as características quantitativas, segundo Müller (1980).

Peso de carcaça quente (PCA): peso de carcaça determinado (em $\mathrm{kg}$ ) logo após o abate, antes de a carcaça entrar na câmara de resfriamento.

Tabela 1 - Composição química dos alimentos e da ração e composição percentual (\%/MS) da dieta experimental Table 1 - Chemical composition of the ingredients and the diet and ingredient composition (\%/DM) of the diet

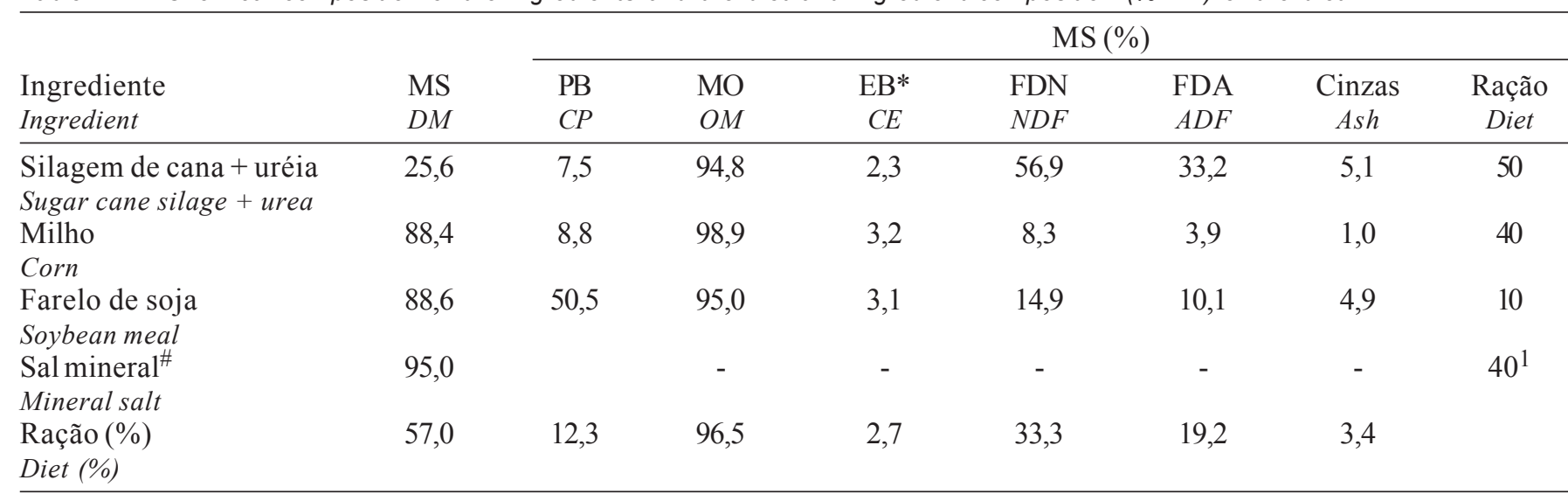

Dados obtidos do Laboratório de Análises de Alimentos e Alimentação Animal do Departamento de Zootecnia/UEM.

${ }^{*}$ Megacalorias $/ \mathrm{kg}$ (megacalories $/ \mathrm{kg}$ ).

\# Níveis de garantia por kg de sal mineral: $130 \mathrm{~g} \mathrm{Ca}, 65 \mathrm{~g} \mathrm{P}, 12 \mathrm{~g} \mathrm{~S}, 12 \mathrm{~g} \mathrm{Mg}, 1500$ mg Fe, 1170 mg Mn, 1300 mg Cu, 71 mg Co, 71 mg I, $21 \mathrm{mg} \mathrm{Se}, 3200 \mathrm{mg} \mathrm{Zi}, 138 \mathrm{~g} \mathrm{Na}, 650 \mathrm{mg} \mathrm{FI}$.

$1 \mathrm{~g} /$ animal/dia (g/animal/day).

MS - matéria seca, PB - proteína bruta, MO - matéria orgânica, EB - energia bruta, FDN - fibra em detergente neutro, FDA - fibra em detergente neutro.

Data obtained from the Laboratory of Feed Analyses and Animal Nutrition of UEM.

\# Level of guarantee per kg of mineral salt: $130 \mathrm{~g} \mathrm{Ca}, 65 \mathrm{~g} \mathrm{P}, 12 \mathrm{~g} \mathrm{~S}, 12 \mathrm{~g} \mathrm{Mg}, 1500 \mathrm{mg}$ Fe, $1170 \mathrm{mg} \mathrm{Mn}, 1300 \mathrm{mg} \mathrm{Cu}, 71 \mathrm{mg}$ Co, $71 \mathrm{mg}$ I, $21 \mathrm{mg} \mathrm{Se}, 3200 \mathrm{mg}$ $\mathrm{Zi}, 138 \mathrm{~g} \mathrm{Na}, 650 \mathrm{mg} \mathrm{Fl}$.

$D M$ - dry matter, CP - crude protein, OM - organic matter, CE - crude energy, NDF - neutral detergent fiber, ADF - acid detergent fiber. 
Peso de carcaça, em arroba (PAR): peso de carcaça determinado em $\mathrm{kg}$ logo após o abate, antes de a carcaça entrar na câmara de resfriamento, dividido por 15 (forma mais utilizada na comercialização de bovinos no Brasil).

Conformação de carcaça (CONF): avaliação realizada subjetivamente segundo escala de pontos apresentada na Tabela 2, em que os valores mais elevados correspondem à melhor conformação. Nesta avaliação é considerado o desenvolvimento muscular, objetivando-se excluir a gordura de cobertura.

Comprimento de carcaça (CC): distância do bordo cranial do osso púbis até o bordo anterior da primeira costela, medida com trena ou fita métrica.

Comprimento da perna (CP): distância entre o bordo anterior do osso do púbis e um ponto médio dos ossos da articulação do tarso, estimada com o auxílio de um compasso de madeira com pontas metálicas. Na seqüência, mediu-se esta distância com o auxílio de uma trena ou fita métrica.

Espessura do coxão (EC): distância obtida com um compasso de madeira com pontas metálicas, entre a face lateral e a medial da porção superior do coxão, que, posteriormente, foi medido com o auxílio de uma trena ou fita métrica.

Área de olho de lombo (AL): no lado direito da carcaça, procedeu-se a um corte transversal entre a $12^{\mathrm{a}}$ e $13^{\mathrm{a}}$ costelas, expondo-se o músculo Longissimus dorsi. Em seguida, foi traçado o seu contorno em papel vegetal e, posteriormente, esta área foi medida com auxílio de um planímetro.

Espessura de gordura subcutânea (EGC): na região do corte entre $12^{\mathrm{a}}$ e $13^{\mathrm{a}}$ costelas, acima do músculo Longissimus dorsi, com o auxílio de um paquímetro, obteve-se a EGC, formada pela média de três pontos de medidas.
Porcentagens de músculo (PM), osso (PO) e gordura (PG) na carcaça: utilizando-se a secção do lombo (Longissimus dorsi), correspondente à $10^{\mathrm{a}}$, $11^{\mathrm{a}}$ e $12^{\mathrm{a}}$ costelas, cujo corte foi obtido segundo o método de Hankins \& Howe (1946), realizou-se a separação física de músculo, osso e gordura e a pesagem individual destes componentes. As respectivas porcentagens obtidas nesta secção foram colocadas nas equações de regressão propostas por Müller (1980), descritas a seguir, transformando os dados correspondentes à $10^{\mathrm{a}}, 11^{\mathrm{a}}$ e $12^{\mathrm{a}}$ costelas:

$$
\begin{aligned}
& \% \mathrm{M}=6,292+0,910 \mathrm{X}_{1} \\
& \% \mathrm{O}=2,117+0,860 \mathrm{X}_{2} \\
& \% \mathrm{G}=1,526+0,913 \mathrm{X}_{3}
\end{aligned}
$$

em que: $X_{i}$ representa, respectivamente, os percentuais de músculo, osso e gordura.

Os percentuais correspondentes à $10^{\mathrm{a}}, 11^{\mathrm{a}}$ e $12^{\mathrm{a}}$ costelas foram colocados nas equações de regressão, segundo o método de Hankins \& Howe (1946), abaixo citadas, obtendo-se os percentuais de músculo (PM), osso (PO) e gordura (PG) nas carcaças estudadas:

$$
\begin{aligned}
& \mathrm{PM}=15,56+0,81 \mathrm{M} \\
& \mathrm{PO}=4,30+0,61 \mathrm{O} \\
& \mathrm{PG}=3,06+0,82 \mathrm{G}
\end{aligned}
$$

em que: $\mathrm{M}$, $\mathrm{O}$ e $\mathrm{G}$ representam, respectivamente, os valores de músculo, osso e gordura encontrados pelas equações propostas por Muller (1980).

Marmoreio (MAR): gordura intramuscular observada no músculo Longissimus dorsi, entre a $12^{\mathrm{a}}$ e $13^{\mathrm{a}}$ costelas, conforme pontuação apresentada na Tabela 3 .

Textura (TXT): determinada pelo tamanho dos fas-

\begin{tabular}{|c|c|c|c|c|c|c|c|}
\hline $\begin{array}{l}\text { Conformação } \\
\text { Conformation }\end{array}$ & $\begin{array}{l}\text { Mais } \\
\text { Plus }\end{array}$ & $\begin{array}{l}\text { Média } \\
\text { Mean }\end{array}$ & $\begin{array}{c}\text { Menos } \\
\text { Minus }\end{array}$ & $\begin{array}{l}\text { Conformação } \\
\text { Conformation }\end{array}$ & $\begin{array}{l}\text { Mais } \\
\text { Plus }\end{array}$ & $\begin{array}{l}\text { Média } \\
\text { Mean }\end{array}$ & $\begin{array}{c}\text { Menos } \\
\text { Minus }\end{array}$ \\
\hline $\begin{array}{l}\text { Superior } \\
\text { Superior }\end{array}$ & 18 & 17 & 16 & $\begin{array}{l}\text { Regular } \\
\text { Regular }\end{array}$ & 9 & 8 & 7 \\
\hline $\begin{array}{l}\text { Muito boa } \\
\text { Very good }\end{array}$ & 15 & 14 & 13 & $\begin{array}{l}\text { Má } \\
\text { Poor }\end{array}$ & 6 & 5 & 4 \\
\hline $\begin{array}{l}\text { Boa } \\
\text { Good }\end{array}$ & 12 & 11 & 10 & $\begin{array}{l}\text { Inferior } \\
\text { Inferior }\end{array}$ & 3 & 2 & 1 \\
\hline
\end{tabular}
cículos (grãos de carne) e avaliada subjetivamente, por meio de uma escala de pontos (Tabela 4). Foi observada no mesmo local utilizado para avaliação do marmoreio.

Tabela 2 - Sistema de pontuação para a avaliação da conformação de carcaças Table 2 - Score system for evaluation of carcass conformation

Fonte (Source): Müller (1980). 
Coloração (COR): coloração apresentada pelo músculo após resfriamento das carcaças por 24 horas. Realizou-se um corte transversal do músculo Longissimus dorsi, na região entre a $12^{\mathrm{a}}$ e $13^{\mathrm{a}}$ costelas e, após 30 minutos, fez-se a avaliação segundo a escala de pontuação (Tabela 4).

Perdas ao descongelamento e à cocção, maciez, suculência e palatabilidade da carne: a porção do músculo Longissimus dorsi entre a $10^{\mathrm{a}}$ e $12^{\mathrm{a}}$ costelas foi retirada das carcaças, acondicionadas em sacos plásticos identificados com o tratamento e animal e congelada, para posterior análise. Destas amostras, utilizando-se da metodologia descrita por Moletta(1990), foram coletados bifes de 2,5 cm de espessura, pesados, descongelados em refrigerador, por 24 horas, e pesados novamente, para obtenção das perdas pelo descongelamento (PDE). Em seguida, os bifes foram assados por 15 minutos a uma temperatura de $70^{\circ} \mathrm{C}$. Ao serem retirados do forno, procedeu-se à nova pesagem, para cálculo das perdas pela cocção da carne.

Cada amostra (bife) foi cortada, ainda quente, em cubos de aproximadamente $2,5 \mathrm{~cm}^{3}$ e distribuídos ao acaso para um "painel" composto por cinco avaliadores treinados que avaliaram subjetivamente a maciez, suculência e palatabilidade da carne, atribuindo valores conforme a escala de pontuação apresentada na Tabela 5 .

O delineamento experimental foi inteiramente casualizado, com três tratamentos e seis repetições para o tratamento PRC e sete para os tratamentos VAZ e CHU. Os dados de peso de carcaça, em kg e em arrobas, de comprimentos de carcaça e de perna, espessuras do coxão e gordura, proporção de osso, músculo e gordura, cor, textura, marmoreio, perdas ao descongelamento e à cocção, maciez, suculência e palatabilidade foram submetidos à análise de variância e as médias foram comparadas pelo teste Tukey a $5 \%$ de significância, utilizando-se o SAEG (1997), conforme o seguinte modelo:

$$
Y_{i j}=\mu+t_{i}+e_{i j}
$$

em que: $Y_{i j}=$ observação no animal $\mathrm{j}$ submetido ao tratamento $\mathrm{i} ; \mu=$ constante geral; $\mathrm{t}_{\mathrm{i}}=$ efeito do tratamento $\mathrm{i} ; \mathrm{i}=1 ; \ldots ; 3 ; \mathrm{e}_{\mathrm{ij}}=$ erro aleatório associado a cada observação $Y_{i j}$.

Tabela 3 - Escala de pontos para avaliação do grau de marmoreio

Table 3 - Score for evaluation of marbling grade

\begin{tabular}{|c|c|c|c|c|c|c|c|}
\hline $\begin{array}{l}\text { Marmoreio } \\
\text { Marbling }\end{array}$ & $\begin{array}{l}\text { Mais } \\
\text { Plus }\end{array}$ & $\begin{array}{l}\text { Médio } \\
\text { Mean }\end{array}$ & $\begin{array}{l}\text { Menos } \\
\text { Minus }\end{array}$ & $\begin{array}{c}\text { Marmoreio } \\
\text { Marbling }\end{array}$ & $\begin{array}{l}\text { Mais } \\
\text { Plus }\end{array}$ & $\begin{array}{l}\text { Médio } \\
\text { Mean }\end{array}$ & $\begin{array}{l}\text { Menos } \\
\text { Minus }\end{array}$ \\
\hline $\begin{array}{l}\text { Abundante } \\
\text { Abundant }\end{array}$ & 18 & 17 & 16 & $\begin{array}{l}\text { Pequeno } \\
\text { Small }\end{array}$ & 9 & 8 & 7 \\
\hline $\begin{array}{l}\text { Moderado15 } \\
\text { Moderate }\end{array}$ & 14 & 13 & $\begin{array}{l}\text { Leve } \\
\text { Light }\end{array}$ & 6 & 5 & 4 & \\
\hline $\begin{array}{l}\text { Médio } \\
\text { Mean }\end{array}$ & 12 & 11 & 10 & $\begin{array}{l}\text { Traços } \\
\text { Traces }\end{array}$ & 3 & 2 & 1 \\
\hline
\end{tabular}

Fonte (Source): Müller (1980).

Tabela 4 - Escala de pontos para avaliação da textura e da coloração da carne

Table 4 - Score for evaluation of meat texture and color

\begin{tabular}{|c|c|c|c|}
\hline $\begin{array}{l}\text { Textura } \\
\text { Texture }\end{array}$ & $\begin{array}{l}\text { Pontos } \\
\text { Score }\end{array}$ & $\begin{array}{l}\text { Coloração } \\
\text { Coloration }\end{array}$ & $\begin{array}{l}\text { Pontos } \\
\text { Score }\end{array}$ \\
\hline Muito fina & 5 & Vermelho viva & 5 \\
\hline Very thin & & Cherry red & \\
\hline Fina & 4 & Vermelha & 4 \\
\hline Thin & & Red & \\
\hline $\begin{array}{l}\text { Levemente grosseira } \\
\text { Sligtly coarse }\end{array}$ & a 3 & $\begin{array}{c}\text { Vermelha levemente escura } \\
\text { Ligtly dark red }\end{array}$ & a 3 \\
\hline Grosseira & 2 & Vermelho escura & 2 \\
\hline Coarse & & Dark red & \\
\hline $\begin{array}{l}\text { Muito grosseira } \\
\text { Very coarse }\end{array}$ & 1 & $\begin{array}{l}\text { Escura } \\
\text { Dark }\end{array}$ & 1 \\
\hline
\end{tabular}

Fonte (Source): Müller (1980).

\section{Resultados e Discussão}

Não houve diferença para PVA, PCA e PAR entre as novilhas dos tratamentos VAZ $(422,6 ; 207,1$ e 13,8$)$, CHU $(414,8 ; 200,1$ e 13,3$)$ e PRC $(451,8$; 219,6 e 14,6), conforme se observa na Tabela 6.

O valor médio encontrado para PVA $(429,8 \mathrm{~kg})$ das búfalas pode ser considerado elevado para esta idade e categoria animal, pois Jorge \& Fontes (2001) obtiveram peso de $358,4 \mathrm{~kg}$ em fêmeas terminadas em pastagens aos 24 meses. Rodrigues et al. (2003), avaliando a terminação de machos bubalinos castrados e não-castrados aos 22 meses, observaram peso de 425,5 e 449,5, respectivamen- 
Tabela 5 - Escala de pontuação utilizada pelo painel, para avaliação da maciez, suculência e palatabilidade da carne Table 5 - Score of points used for evaluation of tenderness, juiciness and flavor of the meat

\begin{tabular}{lcc}
\hline Maciez & Suculência & Palatabilidade \\
Tenderness & Juiciness & Flavor \\
\hline Extremamente macia & Extremamente suculenta & Extremamente saborosa \\
Extremely tender & Extremelyjuicy & Extremely tasty \\
Muito macia & Muito suculenta & Muito saborosa \\
Verytender & Veryjuicy & Very tasty \\
Macia & Suculenta & Saborosa \\
Tender & Juicy & Tasty \\
Levemente acima da média & Levemente acima da média & Levemente acima da média \\
Slightly up mean & Slightly up mean & Slightly up mean \\
Média & Média & Média \\
Mean & Mean & Mean \\
Levemente abaixo da média & Levemente abaixo da média & Levemente abaixo da média \\
Slightly down mean & Slightly down mean & Slightly down mean \\
Dura & Pouco suculenta & Pouco saborosa \\
Firm & Littlejuicy & Little tasty \\
Muito dura & Muito pouco suculenta & 6 \\
Very firm & Verylittlejuicy & 5 \\
Extremamente dura & Sem suculência & Muito pouco saborosa \\
Extremelyfirm & Withoutjuiciness & Verylittle tasty \\
\hline Fon & Sem sabor
\end{tabular}

Fonte (Source): Muller (1980).

Tabela 6 - Peso vivo ao abate (PVA), peso de carcaça quente (PCA) e peso de carcaça, em arrobas (PAR), de novilhas bubalinas em diferentes condições reprodutivas, terminadas em confinamento.

Table 6 - Slaughter body weight (SBW), hot carcass weight (HCW) and carcass weight - @ (CW) of buffalo heifers in different reproductive conditions finished in feedlot

\begin{tabular}{lcccc}
\hline $\begin{array}{l}\text { Parâmetro } \\
\text { Item }\end{array}$ & $\begin{array}{c}\mathrm{VAZ}^{1} \\
N P^{1}\end{array}$ & $\begin{array}{c}\mathrm{CHU}^{2} \\
L^{2}\end{array}$ & $\begin{array}{c}\mathrm{PRC}^{3} \\
G P^{3}\end{array}$ & $\mathrm{CV}(\%)$ \\
\hline PVA, kg & 422,6 & 414,8 & 451,8 & 9,0 \\
SBW, kg & & & & \\
PCA, kg & 207,1 & 200,1 & 219,6 & 9,4 \\
HCW, kg & & & & \\
PAR, @ & 13,8 & 13,3 & 14,6 & 9,4 \\
CW, @ & & & &
\end{tabular}

${ }^{1} \mathrm{VAZ}$ - Novilhas vazias.

${ }^{2} \mathrm{CHU}$ - Novilhas com chumbo no útero.

${ }^{3}$ PRC - Novilhas com implante hormonal.

${ }^{1} \mathrm{NP}$ - No pregnant heifers.

${ }^{2} L-$ Lead spheres in the uterine corn.

${ }^{3} \mathrm{GP}$ - hormone implanted heifers.

Coeficiente de variação (Coefficient of variation).

te, demonstrando que a condição reprodutiva não interferiu nesta característica.

O PCA é uma característica importante, pois está diretamente relacionado ao valor comercial da carcaça. Os valores de PCA dos três tratamentos deste experimento foram de $209,0 \mathrm{~kg}$, superiores ao obtido por Jorge \& Fontes (2001), de 196,1 kg, em fêmeas bubalinas terminadas em pastagens aos 24 meses.
Rodrigues et al. (2003), avaliando a terminação de machos bubalinos castrados e não-castrados aos 22 meses, observaram PCA de 246,6 e 261,8, respectivamente, sendo significativamente superior para os animais inteiros, e ambos superiores aos deste ensaio, confirmando o melhor peso de carcaça de machos em relação a fêmeas de mesma idade.

OPAR, que neste trabalho foi, em média, de 13,9@, é uma característica que acompanha o PCA, pois é o dividendo desta variável por 15. A importância desta informação está ligada à forma de remuneração do animal ao produtor. $\mathrm{O}$ valor mínimo do PAR é muito variável entre as regiões no Brasil, podendo ser aceitas desde10@,na região Sul, até12@, nas regiões Centro-Oeste e Norte; abaixo destes valores pode haver deságio na comercialização de carcaças de fêmeas (ANUALPEC, 2003). Os valores deste ensaio (Tabela 6) foram superiores ao mínimo exigido, atendendo, portanto, as exigências de mercado.

Os dados de conformação de carcaça (CONF), comprimentos de carcaça (CC) e de perna (CP) e espessura do coxão (EC) de novilhas bubalinas em diferentes condições fisiológicas terminadas em confinamento estão apresentados na Tabela 7.

Não houve diferença $(\mathrm{P}>0,05)$ entre os tratamentos para as variáveis CONF, CC, CP e EC. Neste estudo, a conformação nos três tratamentos foi regular média, demonstrando que caberia maior proporção 
Tabela 7 - Conformação de carcaça (CONF), comprimento de carcaça (CC), comprimento de perna (CP) e espessura do coxão (EC) de novilhas bubalinas em diferentes condições fisiológicas terminadas em confinamento

Table 7 - Carcass conformation (CONF), carcass length $(C L)$, leg length (LL) and beef round thickness (BRT) of buffalo heifers in different physiologic condition finished in feedlot

\begin{tabular}{lcccc}
\hline $\begin{array}{l}\text { Parâmetro } \\
\text { Parameter }\end{array}$ & $\begin{array}{c}\mathrm{VAZ}^{1} \\
N P^{1}\end{array}$ & $\begin{array}{c}\mathrm{CHU}^{2} \\
L^{2}\end{array}$ & $\begin{array}{c}\mathrm{PRC}^{3} \\
G P^{3}\end{array}$ & $\mathrm{CV}(\%)$ \\
\hline $\begin{array}{l}\text { CONF, pontos } \\
\text { COM, score }\end{array}$ & 9,1 & 7,6 & 7,8 & 24,8 \\
$\begin{array}{l}\text { CC, cm } \\
C L, c m\end{array}$ & 124,1 & 123,1 & 121,5 & 3,0 \\
CP, cm & 69,8 & 67,4 & 67,7 & 3,0 \\
$L L, c m$ & 25,3 & 25,3 & 24,4 & 4,7 \\
$\begin{array}{l}\text { EC }, \mathrm{cm} \\
\text { BRT, } \mathrm{cm}\end{array}$ & & & & \\
\hline
\end{tabular}

${ }^{1} \mathrm{VAZ}$ - Novilhas vazias.

${ }^{2} \mathrm{CHU}$ - Novilhas com chumbo no útero.

${ }^{3}$ PRC - Novilhas com implante hormonal.

${ }^{1} N P$ - No pregnant heifers.

${ }^{2} L-$ Lead spheres in the uterine corn.

3 GP-hormone implanted heifers.

Coeficiente de variação (Coefficient of variation).

de músculos para uma carcaça mais convexa, de maior valor comercial.

Todavia, este valor foi superior ao encontrado por Rodrigues et al. (2001), que, utilizando machos inteiros com 24 meses da raça Jafarabadi, estimaram por ultra-som CONF de 3,3 pontos. No entanto, os valores obtidos neste estudo foram semelhantes ao valor de 9,0 pontos obtidos por Vaz et al. (2003), em bubalinos da raça Mediterrâneo terminados em confinamento com peso de abate de $362 \mathrm{~kg}$ e alimentados com silagem de milho ou cana-de-açúcar como volumoso. A CONF é uma característica subjetiva utilizada para avaliação da musculosidade em bovinos e, desta forma, valores não muito expressivos são esperados para bubalinos, como demonstrado pelos valores deste trabalho.

O CC deste experimento foi de $122,9 \mathrm{~cm}$, que foi inferior ao valor de $130 \mathrm{~cm}$ encontrado por Jorge et al. (1997). Porém, esses autores trabalharam com machos Mediterrâneos mais velhos que os deste traba1ho. Da mesma forma, foi inferior ao valor encontrado por Jorge et al. (2003b), que abateram machos das raças Murrah, Jafarabadi e Mediterrâneo com 18 meses de idade e diferentes estádios de maturidade, ou seja, 400, 450 e $500 \mathrm{~kg}$ e obtiveram CC de 134; 138 e $141 \mathrm{~cm}$, respectivamente. No entanto, o valor obtido neste estudo foi superior ao encontrado por Vaz et al.
(2003), em machos da raça Mediterrâneo terminados em confinamento e abatidos aos $362 \mathrm{~kg}$.

O CP médio dos três tratamentos foi de $68,1 \mathrm{~cm}$, que foi maior que aquele registrado por Vaz et al. (2003), de 63,5 cm, em bubalinos da raça Mediterrâneo terminados em confinamento.

A EC média foi de $25,0 \mathrm{~cm}$, inferior à obtida por Rodrigues et al. (2001), em machos Jafarabadi aos 24 meses, avaliados por ultra-som $(43,1 \mathrm{~cm})$. No entanto, o valor deste trabalho foi superior ao estimado por Vaz et al. (2003-21,1 cm), em búfalos da raça Mediterrâneo terminados em confinamento alimentados com silagem de milho ou cana-de-açúcar. Por outro lado, a EC foi inferior à encontrada por Jorge et al. (2003a), que abateram machos das raças Murrah, Jafarabadi e Mediterrâneo com 18 meses de idade e diferentes estágios de maturidade, ou seja, 400, 450 e $500 \mathrm{~kg}$, e obtiveram EC de 27,7; 26,9 e 25,7 cm, respectivamente. $\mathrm{A} E \mathrm{E}$, como a conformação, está ligada à musculosidade e, portanto, é influenciada pelos mesmos fatores.

Os valores médios para AOL, AOC e EGC não foram influenciados pelos tratamentos (Tabela 8).

A AOL é um indicador importante da musculosidade da carcaça e aumenta com o peso da carcaça. Os valores obtidos neste trabalho (médio = $57,1 \mathrm{~cm}^{2}$ ) foram superiores ao valor encontrado por Jorge et al. (1997), que, trabalhando com machos Mediterrâneo com 24 meses de idade em confinamento, obtiveram AOL de $43,9 \mathrm{~cm}^{2}$. Foram superiores também ao valor estimado $\left(45,8 \mathrm{~cm}^{2}\right)$ via ultra-som, por Rodrigues et al. (2001), em machos Jafarabadi aos 24 meses de idade e aos valores encontrados por Jorge \& Fontes (2001), em fêmeas terminadas em pastagens aos 24 meses $\left(41,8 \mathrm{~cm}^{2}\right)$.

A AOL foi superior ainda ao valor médio registrado por Jorge et al. (2003b), de 55,6 $\mathrm{cm}^{2}$, ao trabalharem com diferentes grupos genéticos de bubalinos em diferentes estágios de maturidade. No entanto, foi inferior ao valor estimado $\left(62,6 \mathrm{~cm}^{2}\right)$ por Calixto et al. (2003), em machos Mediterrâneos em diferentes estádios de maturidade.

A AOL média, quando ajustada para $100 \mathrm{~kg}$ de peso vivo (AOC), foi de $27,6 \mathrm{~cm}^{2}$, superior à observada por Jorge et al. $\left(1997-17,8 \mathrm{~cm}^{2}\right)$, em búfalos machos de 24 meses de idade, e por Jorge et al. (2003b), de $23,7 \mathrm{~cm}^{2}$, em bubalinos de diversos grupos genéticos em diferentes estádios de maturidade.

Segundo Felício (1993), a EGC é um indicador de qualidade da carcaça, por apontar o tipo de alimento 
recebido pelo animal (alta ou baixa energia) e por alterar diretamente a velocidade de resfriamento da carcaça, comportando-se como isolante térmico. Em ambas as situações, a maciez da carne é o parâmetro mais afetado, e sempre positivamente.

A EGC não diferiu entre os tratamentos, embora se esperasse que os animais com o uso de promotor apresentassem espessura de gordura de cobertura menor, em função da ação anabolizante, que proporciona maior deposição de tecido muscular, como constatado por Reiling et al. (1996). A EGC (4,8 mm) obtida está acima do mínimo exigido pelo mercado (3,0 mm), conforme citado por Costa et al. (2002). Porém, foi inferior às EGC encontradas por Jorge et al. (1997), que obtiveram 5,9 mm em bubalinos machos aos 24 meses, e por Jorge \& Fontes (2001), em fêmeas terminadas em pastagens aos 24 meses $(7,4 \mathrm{~mm})$. Todavia, foi superior à estimada $(2,8 \mathrm{~mm})$ por Rodrigues et al. (2001), em machos inteiros Jafarabadi aos 24 meses de idade, e por Jorge et al. (2003b) que abateram animais das raças Murrah, Jafarabadi e Mediterrâneo com pesos de 400; 450 e $500 \mathrm{~kg}$ e obtiveram 2,2; 3,0 e 4,0 mm de EGC, respectivamente. Todavia, Calixto et al. (2003) estimaram, em animais da raça Mediterrânea com pesos de 450; 480; 510 e $540 \mathrm{~kg}$, respectivamente, EGC de 5,8; 6,9; 7,0 e 7,0 mm, que foram superiores aos valores deste trabalho. Possivelmente, esta superioridade esteja ligada à alimentação e ao peso final dos animais, que foi mais elevado.

Tabela 8 - Área de olho de lombo (AOL), área de olho de lombo por $100 \mathrm{~kg}$ de carcaça (AOC) e espessura de gordura de cobertura (EGC) de noviIhas bubalinas em diferentes condições fisiológicas terminadas em confinamento

Table 8 - "Longissimus dorsi" area (LYA), Longissimus dorsi area per $100 \mathrm{~kg}$ of carcass (LYA\%) and subcutaneous fat thickness (FAT) of buffalo heifers in different physiologic conditions finished in feedlot

\begin{tabular}{lcccc}
\hline Parâmetro & $\begin{array}{c}\mathrm{VAZ}^{1} \\
\text { Item }\end{array}$ & $\begin{array}{c}\mathrm{CHU}^{2} \\
L^{2}\end{array}$ & $\begin{array}{c}\mathrm{PRC}^{3} \\
G P^{3}\end{array}$ & ${ }^{*} \mathrm{CV}(\%)$ \\
\hline $\mathrm{AOL}, \mathrm{cm}^{2}$ & 58,1 & 56,5 & 56,6 & 9,1 \\
LYA $\mathrm{cm}^{2}$ & & & & \\
$\mathrm{AOC}, \mathrm{cm}^{2}$ & 28,2 & 28,5 & 26,0 & 14,0 \\
LYA\%, $\mathrm{cm}^{2}$ & 5,6 & 4,5 & 4,5 & 35,4 \\
EGC, $\mathrm{mm}$ & & & & \\
FAT, $m \mathrm{~mm}$ & & & &
\end{tabular}

${ }^{1} \mathrm{VAZ}$ - Novilhas vazias.

${ }^{2} \mathrm{CHU}$ - Novilhas com chumbo no útero.

${ }^{3}$ PRC - Novilhas com implante hormonal.

${ }^{1} \mathrm{NP}$ - No pregnant heifers.

${ }^{2} L-$ Lead spheres in the uterine corn.

${ }^{3} \mathrm{GP}$ - hormone implanted heifers.

Coeficiente de variação (Coefficient of variation).
Não houve diferença significativa para as variáveis PM, PO, PG, relação músculo/gordura e relação músculo mais gordura (porção comestível)/osso, para as búfalas dos tratamentos VAZ, CHU e PRC (Tabela 9). A não-ocorrência de diferenças entre PM e PG não era esperada, pois os animais do tratamento PRC deveriam apresentar PM maior e PG menor que os dos demais tratamentos, em função do efeito anabolizante do promotor, que proporciona maior deposição de tecido muscular em detrimento ao adiposo, conforme relatado por Reiling et al. (1996).

O percentual médio para PM foi de $60,6 \%$, semelhante ao encontrado por Jorge \& Fontes (2001), em fêmeas da raça Mediterrânea terminadas em pastagens aos 24 meses. Esta porcentagem foi superior às obtidas por Jorge et al. (1997), de 55,9\%, em machos Mediterrâneos abatidos aos 24 meses de idade, e por Jorge et al. (2003b) que abateram animais das raças Murrah, Jafarabadi e Mediterrânea com pesos de $400 ; 450$ e $500 \mathrm{~kg}$ e obtiveram 56,$3 ; 54,7$ e $55,7 \%$, respectivamente. Entretanto, foi inferior às estimadas por Calixto et al. (2003), em machos da raça Mediterrânea com pesos de $450 ; 480 ; 510$ e $540 \mathrm{~kg}(54,1 ; 53,7$; 50,2 e $52,2 \%$, respectivamente). Todavia, foi inferior ao valor encontrado por Morgan (1993), que, citando vários trabalhos envovendo bubalinos, observaram média de $65,4 \%$.

A PO média foi de $18,9 \%$, superior aos valores citados por Morgan (1993), a partir de vários trabalhos, em que a PO média foi de 18,3\%. Do mesmo modo, Jorge et al. (1997) obtiveram PO de 16,5\%. Jorge et al. (2003b) abateram animais das raças Murrah, Jafarabadi e Mediterrânea com pesos de 400; 450 e $500 \mathrm{~kg}$ e observaram PO de 27,2;29,1 e $30,2 \%$, respectivamente. Calixto et al. (2003), por sua vez, em machos da raça Mediterrânea com 450; 480; 510 e $540 \mathrm{~kg}$, observaram PO de 16,$9 ; 15,9 ; 15,8$ e $15,6 \%$, respectivamente.

A PG média foi de $20,5 \%$. Todavia, Jorge et al. (1997) observaram maior PG na carcaça de bubalinos (27,6\%). Calixto et al. (2003), trabalhando com machos da raça Mediterrânea com pesos de 450; $480 ; 510$ e $540 \mathrm{~kg}$, observaram PG de 29,5; 31,4; 34,9 e 33,3\%, respectivamente. Por outro lado, Morgan (1993), revisando vários trabalhos, calcularam média de 16,2\% de PG. Da mesma forma, Jorge et al. (2003b), ao abaterem animais das raças Murrah, Jafarabadi e Mediterrânea com pesos de 400; 450 e 500 kg, obtiveram 16,$5 ; 16,1$ e $14,0 \%$, respectivamente. 
Tabela 9 - Porcentagens de músculo (PM), de osso (PO), de gordura $(P G)$, relações músculo/osso e músculo + gordura/osso, em carcaças de novilhas bubalinas em diferentes condições fisiológicas, terminadas em confinamento

Table 9 - Muscle percentage (MP), bone percentage (BP), fat percentage (FP), muscle/bone and muscle + fat/ bone ratios in carcass of buffalo heifers in different physiologic conditions finished in feedlot

\begin{tabular}{|c|c|c|c|c|}
\hline $\begin{array}{l}\text { Parâmetro } \\
\text { Item }\end{array}$ & $\begin{array}{l}\mathrm{VAZ}^{1} \\
N P^{l}\end{array}$ & $\begin{array}{c}\mathrm{CHU}^{2} \\
L^{2}\end{array}$ & $\begin{array}{c}\mathrm{PRC}^{3} \\
G P^{3}\end{array}$ & ${ }^{*} \mathrm{CV}(\%)$ \\
\hline $\begin{array}{l}\mathrm{PM}, \% \\
M P, \%\end{array}$ & 59,2 & 61,0 & 61,7 & 4,3 \\
\hline $\begin{array}{l}\mathrm{PO}, \% \\
B P, \%\end{array}$ & 18,7 & 19,1 & 18,8 & 5,5 \\
\hline $\begin{array}{l}\mathrm{PG}, \% \\
\mathrm{FP}, \%\end{array}$ & 22,2 & 19,9 & 19,5 & 16,0 \\
\hline $\begin{array}{l}\text { Relação músculo./osso } \\
\text { Muscle/bone relation }\end{array}$ & 3,2 & 3,2 & 3,3 & - \\
\hline $\begin{array}{l}\text { Rel. músc. + gord./osso } \\
\text { Muscle }+ \text { fat/bone relation }\end{array}$ & 4,4 & 4,2 & 4,3 & - \\
\hline
\end{tabular}

${ }^{1} \mathrm{VAZ}$ - Novilhas vazias.

${ }^{2} \mathrm{CHU}$ - Novilhas com chumbo no útero.

${ }^{3}$ PRC - Novilhas com implante hormonal.

${ }^{1} \mathrm{NP}$ - No pregnant heifers.

${ }^{2} L-$ Lead spheres in the uterine corn.

${ }^{3} \mathrm{GP}$ - hormone implanted heifers.

Coeficiente de variação (Coefficient of variation).

Segundo Berg \& Buterfield (1976), o músculo é o tecido mais importante, porque é o mais desejado pelo consumidor. Uma carcaça superior, para qualquer mercado, deve ter quantidade máxima de músculo, mínima de osso e quantidade ótima de gordura variáveis conforme a preferência do consumidor. Neste trabalho, a PM foi superior a $60,6 \%$, a PG próxima de $20,5 \%$ e a EGC foi de $4,8 \mathrm{~mm}$. Portanto, a carcaça apresentou padrão compatível com a exigência do mercado interno.

A relação músculo/osso foi de 3,2, ficando abaixo da normalmente encontrada na literatura. Esta relação foi inferior ao valor calculado por Morgan (1993), a partir de vários trabalhos (3,6). Do mesmo modo, Jorge etal.(1997) obtiveram relação de 3,4 para bubalinos. Jorge et al. (2003b) abateram animais das raças Murrah, Jafarabadi e Mediterrânea com pesos de 400; 450 e $500 \mathrm{~kg}$ e observaram relação de 3,4; 3,4 e 4,0, respectivamente.

A relação músculo+gordura/osso, ou seja porção comestível/osso, foi de 4,3, possivelmente porque os animais apresentaram condições para continuarem crescendo e depositando tecidos moles, o que alteraria significativamente esta relação, apesar de, no momento do abate, apresentarem acabamento de carcaça adequado ( $\mathrm{EGC}=4,8 \mathrm{~mm})$. A porcentagem de gordura $(\mathrm{PG}=20,5 \%)$, porém, foi abaixo do recomendado. Esta relação foi inferior ao valor médio encontrado por Jorge et al. (1997), em búfalos machos abatidos aos 24 meses de idade $(5,1)$ e por Jorge et al. (2003b), que abateram animais das raças Murrah, Jafarabadi e Mediterrânea com pesos de 400; 450 e $500 \mathrm{~kg}$ e obtiveram 5,1; 5,2 e 6,2, respectivamente.

As variáveis perdas ao descongelamento $(\mathrm{PD})$ e à cocção $(\mathrm{PC})$ e perdas da carne congelada após a cocção (CO), maciez (MAC), suculência (SUC) e palatabilidade (PAL) da carne de novilhas búfalas terminadas em confinamento não diferiram entre os tratamentos (Tabela 10).

As PD foram de $9,3 \%$, quase o dobro dos valores encontrados por Vaz et al. (2002), que obtiveram 3,9\% em novilhos Hereford não-castrados e abatidos aos 24 meses de idade. Entretanto, as PC (31,5\%) verificadas por estes autores foram bem superiores às deste trabalho $(23,4 \%)$, resultando em CO superior $(35,5 \%)$ à observada neste estudo $(30,5 \%)$.

As PC foram semelhantes entre os tratamentos resultando, em suculência e palatabilidade semelhantes. Forrest et al. (1979) afirmam que também a gordura influencia a suculência da carne e concluem que a distribuição uniforme de lipídios no músculo não somente favorece a palatabilidade da carne, servindo também como barreira contra a perda de suco muscular durante o cozimento. Estes autores ressaltam a importância da cobertura de gordura como forma de manter a umidade da carne durante o processo de resfriamento, de modo que a palatabilidade está estreitamente ligada à maciez e suculência da carne.

A MAC foi de 7,8 pontos, SUC de 7,0 pontos e PAL de 6,7 pontos, caracterizando a carne como macia, levemente acima da média a suculenta e levemente acima da média a saborosa, respectivamente. Costa et al. (2002) destacam a associação positiva entre palatabilidade e marmoreio, palatabilidade e extrato etéreo, indicando que a gordura contém substâncias flavorizantes que são agradáveis ao paladar. Esses autores observaram que a maciez da carne esteve associada à palatabilidade, indicando que carnes mais macias foram mais palatáveis, assim como a suculência da carne também esteve associada à palatabilidade.

\section{Conclusões}

A utilização de promotor de crescimento e de esferas de chumbo no interior do útero não alterou as características de carcaça e de carne quando compa- 
Tabela 10 - Perdas ao descongelamento (PD) e à cocção (PC), perdas da carne congelada após a cocção (CO), maciez (MAC), suculência (SUC) e palatabilidade (PAL) da carne de novilhas bubalinas em diferentes condições fisiológicas terminadas em confinamento

Table 10 - Thawing (TL), cooking (CL), thawing by cooking losses after thawing (TCL), tenderness (TEN), juiciness (JUI) flavor (FLA) of meat from buffalo heifers in different physiologic conditions finished in feedlot

\begin{tabular}{lcccc}
\hline $\begin{array}{l}\text { Parâmetro } \\
\text { Item }\end{array}$ & $\begin{array}{c}\mathrm{VAZ}^{1} \\
N P^{1}\end{array}$ & $\begin{array}{c}\mathrm{CHU}^{2} \\
L^{2}\end{array}$ & $\begin{array}{c}\mathrm{PRC}^{3} \\
G P^{3}\end{array}$ & $* \mathrm{CV}(\%)$ \\
\hline $\begin{array}{l}\mathrm{PD}, \% \\
\begin{array}{l}T L \% \\
\mathrm{PC}, \%\end{array}\end{array}$ & 9,4 & 9,2 & 9,1 & 1,9 \\
$\begin{array}{l}C L, \% \\
\mathrm{CO}, \%\end{array}$ & 23,3 & 23,8 & 23,2 & 7,1 \\
$\begin{array}{l}\text { TCL, \% } \\
\text { MAC, pontos }\end{array}$ & 30,5 & 30,8 & 30,2 & 7,8 \\
$\begin{array}{l}\text { TEN, score } \\
\text { SUC, pontos }\end{array}$ & 6,9 & 7,8 & 7,6 & 7,1 \\
$\begin{array}{l}\text { JUI, score } \\
\text { PAL, pontos }\end{array}$ & 6,6 & 7,1 & 7,0 & 6,8 \\
FLA, score & & 6,8 & 6,8 & 7,4 \\
\hline
\end{tabular}

$1 \mathrm{VAZ}$ - Novilhas vazias.

${ }^{2} \mathrm{CHU}$ - Novilhas com chumbo no útero.

${ }^{3}$ PRC - Novilhas com implante hormonal.

${ }^{1} \mathrm{NP}$ - No pregnant heifers.

${ }^{2} L-$ Lead spheres in the uterine corn.

${ }^{3} \mathrm{GP}$ - hormone implanted heifers.

Coeficiente de variação (Coefficient of variation).

rado aos animais vazios. Portanto, se estas práticas forem viáveis do ponto de vista econômico, à saúde do consumidor e ao bem-estar dos animais, podem ser aplicadas de bubalinos em confinamento na fase de terminação.

\section{Literatura Citada}

BARUSELLI, P.S.; OLIVEIRA, J.F.S.; MENDES, M.L.M. et al. Diagnóstico da bubalinocultura do Vale do Ribeira. Campinas: 1993. v.94, 16p. (DocumentoTécnico CATI)

BERG, R.T.; BUTTERFIELD, R.M. New concepts of cattle growth. Sydney: Sydney University Press, 1976. 240p.

CALIXTO, M.G.; JORGE, A.M.; CERVIERI, R.C. et al. Composição física e estudo "in vivo"do desenvolvimento dos tecidos de carcaça de bubalinos jovens terminados em confinamento. In: REUNIÃO ANUAL DA SOCIEDADE BRASILEIRA DE ZOOTECNIA, 40., 2003, Santa Maria. Anais... São Paulo: Sociedade Brasileira de Zootecnia/Gmosis, [2003] CD-ROM. Nutrição de Ruminantes.

COSTA, E.C.; RESTLE, J.; VAZ, F.N. et al. Características da carcaça de novilhos Red Angus superprecoces abatidos com diferentes pesos. Revista Brasileira de Zootecnia, v.31, n.1, p.119-128, 2002.

CROWE, M.A.; ENRIGHT, W.J.; SWIFT, P. et al. Growth and estrous behavior of heifers actively immunized against prostaglandin $\mathrm{F}_{2 \alpha}$. Journal of Animal Science, v.73, p.345-352, 1995.

FELÍCIO, P.E. Fatores ante e post-mortem que afetam a qualidade da carne vermelha. In: REUNIÃO ANUAL DA SOCIEDADE BRASILEIRA DE ZOOTECNIA, 30., 1993, Rio de Janeiro. Anais... Rio de Janeiro: Sociedade Brasileira de Zootecnia, p.43-52, 1993.

FERREIRA, J.M.; BARROS, L.A.; PROFÍRIO, T.A. et al. Detecção de resíduos de zeranol e acetato de trembolona em bovinos mestiços, com ELISA. In: CONGRESSO BRASILEIRO DE CIÊNCIA E TECNOLOGIA DE CARNES, 1., 2001, São Pedro. Anais... Campinas: Instituto de Tecnologia de Alimentos, 2001. p.438-439.

FNP Consultoria, ANUALPEC, Anuário da Pecuária Brasileira. São Paulo: 2003 400p.

FORREST, J.C.; ABERLE, E.D.; HEDRICK, H.B. et al. Fundamentos de ciência de la carne. Zaragoza: Acribia, 1979. 342p.

HANKINS, O.G.; HOWE, P.E. Estimation of the composition of beef carcass and cuts. Washington, D.C.: Agriculture Department, 1946. 20p. (Technical Bulletin - USDA, 926)

HOFFMANN, B. Critical assessment on the use of anabolic hormones also exhibiting sexhormone-like activities in ruminant production. In: REUNIÃO ANUAL DA SOCIEDADE BRASILEIRA DE ZOOTECNIA, 35., 1998, Botucatu. Anais... Botucatu: Sociedade Brasileira de Zootecnia, 1998. p.9-34.

JORGE, A.M.; ANDRIGHETTO, C.; CALIXTO, M.G. et al. Características quantitativas de carcaça de bubalinos de três grupos genéticos terminados em confinamento e abatidos em diferentes estágios de maturidade. In: REUNIÃO ANUAL DA SOCIEDADE BRASILEIRA DE ZOOTECNIA, 40., 2003, Santa Maria. Anais... Santa Maria: Sociedade Brasileira de Zootecnia/Gmosis, [2003a] CD-ROM. Nutrição de Ruminantes.

JORGE, A.M.; CALIXTO, M.G.; ANDRIGHETTO, C. et al. Composição física e relação entre os tecidos de carcaça de bubalinos de três grupos genéticos terminados em confinamento e abatidos em diferentes estágios de maturidade. In: REUNIÃO ANUAL DA SOCIEDADE BRASILEIRA DE ZOOTECNIA, 40., 2003, Santa Maria. Anais... São Paulo: Sociedade Brasileira de Zootecnia/Gmosis, [2003b] CD-ROM. Nutrição de Ruminantes.

JORGE, A.M.; FONTES, C.A.A. Composição física da carcaça de bovinos e bubalinos abatidos em diferentes pesos. In: CONGRESSO BRASILEIRO DE CIÊNCIA E TECNOLOGIA DE CARNES, 1., 2001, São Pedro. Anais... Campinas: Instituto de Tecnologia de Alimentos, 2001. p.82-83.

JORGE, A.M.; FONTES, C.A.A.; FREITAS, J.A. et al. Rendimento de carcaça e de cortes básicos de bovinos e bubalinos, abatidos em diferentes estádios de maturidade. Revista Brasileira de Zootecnia, v.26, n.5, p.1048-1054, 1997.

MACEDO, M.P.; VALVAROSI, E.; SOBRINHO, E.B. et al. Comparação das características de carcaça de bubalinos da raça Mediterrâneo machos e fêmeas criados na região Noroeste do estado de São Paulo abatidos aos dois anos de idade. In: CONGRESSO BRASILEIRO DE CIÊNCIA E TECNOLOGIA DE CARNES, 1., 2001, São Pedro. Anais... Campinas: Instituto de Tecnologia de Alimentos, 2001.p.80-81.

MARQUES, J.A.; PRADO, I.N.; ZEOULA, L.M. et al. Avaliação da mandioca e seus resíduos Industriais em substituição ao milho no desempenho de novilhas de corte. Revista Brasileira de Zootecnia, v.29, n.5, p.1528-1536, 2000.

MARQUES, J.A.; PRADO, I.N.; NASCIMENTO, W.G. et al. Avaliação do desempenho de novilhas mestiças em diferentes 
condições reprodutivas confinadas. In: REUNIÃO ANUAL DA SOCIEDADE BRASILEIRA DE ZOOTECNIA, 38., 2001, Piracicaba. Anais... Piracicaba: Sociedade Brasileira de Zootecnia. 2001.

MATTOS, J.C.A. Patrimônio genético do rebanho bubalino brasileiro. São Paulo: Associação Brasileira de Criadores de Búfalos, 1992. 29p.

MOLETTA, J.L.; RESTLE, J. Influencia do grupo genético sobre características qualitativas da carne de novilhos. Revista Brasileira de Zootecnia, v.25, n.5, p.866-875, 1996.

MORGAN, J.B. Carcass development in swamp and river buffalo. In: REUNIÃO ANUAL DA SOCIEDADE BRASILEIRA DE ZOOTECNIA, 30., 1993, Rio de Janeiro. Anais... Rio de Janeiro: Sociedade Brasileira de Zootecnia, 1993. p.71-100.

MÜLLER, L. Normas para avaliação de carcaça e concurso de carcaças de novilhos. 1.ed. Santa Maria: Universidade Federal de Santa Maria, 1980. 31p.

NOGUEIRA, J.R.; BARBOSA, C.; MATTOS, J.C.A et al. Aspectos da eficiência reprodutiva de bualinos das raças Mediterâneo e Jafarabadi. Boletim de Indústria Animal, v.46, p.45-54, 1989.

NATIONAL RESEARCH COUNCIL - NRC. Nutrient requirements of beef cattle. Washington, D.C.: National Academy of Sciences, 1996. 242p.

OLIVEIRA, J.F.S.; BARUSELLI, P.S.; MATTOS, J.C.A. et al. Performance of Murrah buffaloes in Ribeira Valley. In: WORLD BUFFALO CONGESS, 4., 1994, São Paulo. Anais... São Paulo: 1994. v.2, p.43-45.

PÁDUA, J.D.; SAINZ, R.D.; MIYAGI, E.S. et al. Avaliação de desempenho e de características de carcaça de bovinos de corte confinados, inteiros e castrados com e sem Synovex-s ${ }^{\circledR}$. In: CONGRESSO BRASILEIRO DE CIÊNCIA E TECNOLOGIA DE CARNES, 1., 2001, São Pedro. Anais... Campinas: Instituto de Tecnologia de Alimentos, 2001. p.90-91.

PRADO, I.N.; MARITNS, A.S.; ALCALDE, C.R. et al. Desempenho de novilhas alimentadas com rações contendo milho ou casca de mandioca como fonte energética e farelo de algodão ou levedura como fonte protéica. Revista Brasileira de Zootecnia, v.29, n.1, p.278-287, 2000.

PURSLEY, J.R.; MEE, M.O.; WILTBANK, M.C. Synchronization of ovulation in dairy cows using $\mathrm{PGF}_{2 \alpha}$ and GnRH. Theriogenology, v.44, p.915-923, 1995.

REILING, B.A.; BERGER, L.L.; FAULKNER, F.K. et al. Efects of prenatal androgenization, melengestrol acetate and sinovex-H on feedlot performance, carcass and sensory traits of once-calved heifers. Journal of Animal Science, n.74, p.2043-2051, 1996.
RESTLE, J; BRONDANI, I.L.; BERNARDES, R.A.C. O novilho superprecoce. In: RESTLE, J.(Ed.) Confinamento, pastagens e suplementação para a produção de bovinos de corte. Santa Maria: Universidade Federal de Santa Maria, 1999. p.866-875.

RODRIGUES, V.C.; ANDRADE, I.F.; SOUSA, J.C.D. et al. Avaliação da condição corporal de bubalinos e bovinos através do ultra-som. Ciências Agrotécnicas, v.25, n.5, p.11741184, 2001.

RODRIGUES, V.C.; ANDRADE, I.F.; FREITAS, R.T. et al. Rendimento do abate e carcaça de bovinos e bubalinos castrados e inteiros. Revista Brasileira de Zootecnia, v.32, n.3, p.663-671, 2003.

RUMSEY, T.S., HAMMOND, A.C.; ELSSASER, T.H. Responses to an estrogenic growth promoter in beef steers fed varying nutritional regimens. Journal of Animal Science, v.77, p.2865-2872, 1999a.

RUMSEY, T.S., ELSSASER, T.H.; KAHL, S. Performance and digestibilities of beef cattle fed diets suplemented with either soybean meal or roasted soybeans and implanted with synovex. Journal of Animal Science, v.77, p.1631-1637, 1999b.

RUMSEY, T.S., ELSSASER, T.H., KAHL, S. et al. The effect of roasted soybeans in the diet of feedlot steers and synovex-S ear implants on carcass characteristics and estimated composition. Journal of Animal Science, v.77, p.17261734, 1999c.

UNIVERSIDADE FEDERAL DE VIÇOSA - UFV. SAEG Sistema de Análises Estatísticas e Genéticas.Versão 7.1. Viçosa, MG. 1997. 150p. (Manual do usuário).

VAZ, F.N.; RESTLE, J.; QUADROS, A.R.B. et al. Características de carcaça e da carne de novilhos e vacas de descarte Hereford, terminadas em confinamento. Revista Brasileira de Zootecnia, v.31, n.3, p.1501-1510, 2002. (Suplemento).

VAZ, F.N.; RESTLE, J.; BRONDANI, I.L. et al. Estudo da carcaça e da carne de bubalinos mediterrâneo terminados em confinamentocom diferentes fontes de volumosos. Revista Brasileira de Zootecnia, v.32, n.2, p.393-404, 2003.

VILLARES, J.B. Social aspects of buffaloes breeding for social economics conditions progress of the mankind. In: WORLD BUFFAlO CONGRESS, 4., 1994, São Paulo. Anais... São Paulo: 1994. v.1, p.190-195.

Recebido em: 29/06/04 Aceito em: 08/06/05 